\title{
Immunohistochemical Markers of Tumor Microenvironment as Prognostic Predictors in Diffuse Large B-Cell Lymphoma: Study from an Oncology Centre in South India
}

\author{
Indu R. Nair ${ }^{1, \odot ~ A t h u l y a ~ S a d e e s h ~}{ }^{1} \quad$ Pooja Phalak ${ }^{1}$ \\ ${ }^{1}$ Department of Pathology, Amrita Institute of Medical Sciences, \\ Kochi, Kerala, India \\ 2Department of Medical Oncology, Amrita Institute of Medical \\ Sciences, Kochi, Kerala, India
}

Pavithran Keechilat ${ }^{1,2}$

\begin{abstract}
Address for correspondence Indu R. Nair, MD, Department of Pathology, Department of Pathology, Amrita Institute of Medical Sciences, Elamakkara Post Office, Kochi 682041, Kerala, India (e-mail: drinduharikrishnan@gmail.com).
\end{abstract}

Abstract

Keywords
- CD34
- CD68
- DLBCL
- immunohistochem-
istry
- microenvironment
- prognosis

Introduction Diffuse large B-cell lymphoma (DLBCL) accounts for $60 \%$ of lymphomas in India. Although the survival of DLBCL patients has improved following the addition of rituximab, a subset of patients do not respond well to therapy. Among the several factors responsible for this varied response, tumor microenvironment is considered to be crucial. This study is a search for such prognostic markers in the tumor microenvironment.

Materials and Methods A total of 97 patients were selected, of whom 34 were treated with the CHOP regimen and 63 with RCHOP. Immunohistochemistry for CD68 was performed to study the stromal- 1 signature and CD34 for stromal-2 signature.

Results There was a significant increase in the counts of CD68-positive cells among patients free of events. CD34 count was higher in patients with events in both CHOP and RCHOP groups.

Conclusion Additional assessment of stromal microenvironment along with the cell of origin might predict the clinical outcome better in DLBCL.

\section{Introduction}

Diffuse large B-cell lymphoma (DLBCL) accounts for 25 to $30 \%$ of non-Hodgkin lymphoma (NHL) among adults in the west and as high as $60 \%$ of NHL in India. ${ }^{1}$ Although the survival of DLBCL patients has improved following the addition of rituximab to the conventional therapeutic agents, relapse or refractory disease occurs in a subset of patients. As the tumor does not show significant morphological variations, heterogenous biology most likely results in this varied outcome. ${ }^{2}$ Numerous immunohistochemical (IHC) and molecular studies have been conducted on the tumor cells in order to find out this difference in behavior. ${ }^{3}$ Recently, the tumor

published online Jun 16, 2020
DOI https://doi.org/ $10.1055 / \mathrm{s}-0040-1714305$ ISSN 2454-6798. microenvironment has gained importance, as it is suggested that the crosstalk between the tumor cells and the cells in the microenvironment plays a crucial role in the tumor biology by Lenz et al. ${ }^{4} \mathrm{CD} 68$ is among the several markers for macrophages that form a component of stromal- 1 signature. ${ }^{5}$ CD34 stains the endothelial cells and highlights the angiogenesis, which is described as stromal-2 signature. It gives more specific staining than CD31. ${ }^{6}$

\section{Materials and Methods}

It is a retrospective study conducted at the Departments of Pathology and Medical Oncology. (c) 2020. Spring Hope Cancer Foundation \& Young Oncologist Group of Asia.

This is an open access article published by Thieme under the terms of the Creative Commons Attribution-NonDerivative-NonCommercial-License, permitting copying and reproduction so long as the original work is given appropriate credit. Contents may not be used for commercial purposes, or adapted, remixed, transformed or built upon. (https://creativecommons.org/licenses/by-nc-nd/4.0/)

Thieme Medical and Scientific Publishers Pvt. Ltd., A-12, 2nd Floor, Sector 2, Noida-201301 UP, India 
A total of 97 patients diagnosed with DLBCL and treated with either RCHOP or CHOP at our institute during the period 2010 to2016 were selected. The clinical characteristics of each patient were obtained from medical records. Pathological features were assessed from the retrieved histopathology slides. The tissue sections of each patient were obtained, and IHC was performed using antibodies (CD34clone-QBend/10, Biogenex, CD68 Clone-KP-1, and Dako, prediluted). All the patients were followed up.

The patients were divided into two groups based on the treatment received: those treated without rituximab (CHOP) and those treated with rituximab (RCHOP). Of the 97patients, 34 patients were treated with the CHOP regimen and 63 with RCHOP. The events that occurred in these patients in the form of relapse, recurrence, or death were recorded in both treatment groups separately.

For CD34 analysis, a total of five $40 \times$ fields with highest density areas in the representative slide were selected. Photomicrographs of a known area $(n \times 1 / 0.26$ is the area of one high power field [40x] in Zeiss microscope) were taken and analyzed. CD34-positive vessels/cells were counted using ImageJ analysis (Java-based image processing program developed at the National Institutes of Health and the Laboratory for Optical and Computational Instrumentation), ${ }^{7}$ and the mean was calculated and noted as the microvascular density (MVD) ( - Fig. 1).

CD68-positive cells (macrophages) in a total of five fields were selected for analysis. Individual CD68-positive cells were counted using the same method at $400 \times$ magnification,

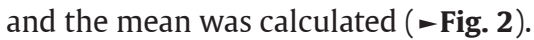

All patients were followed up, and events, if present, were recorded from the electronic medical records.

\section{Results}

A male preponderance was seen in both groups. In the $\mathrm{CHOP}$ arm, $76.5 \%$ were males, and in RCHOP arm, $71.4 \%$ were males. Extranodal disease was seen in $47.1 \%$ of $\mathrm{CHOP}$ and $28.6 \%$ of RCHOP treated patients.

Among the patients treated with the CHOP regimen, events occurred in 12 out of 34 as relapse in 11 and death in 1 (35.3\%). Four patients had events (three relapses and one death) at less than 2 years, three had between 2 to 5 years, and five had after 5 years ( - Fig. $\mathbf{3}$ ).

14 of the 63 patients treated with RCHOP had events (22.2\%), as relapse in 7 , at less than 2 years in 3 , and between 2 and 5 years in the remaining 4 . Deaths of another 7 patients were recorded: five at less than 2 years and two after 5 years ( - Fig. 4 ).

In the RCHOP group with events, the number of stromal cells stained by CD68 ranged from 26.9 to $258 / \mathrm{mm}^{2}$, with a mean of $101.5 / \mathrm{mm}^{2}$. In those without any events, the counts were between 115.9 and $410 / \mathrm{mm}^{2}$, with a mean of $302.1 / \mathrm{mm}^{2}$.

In patients with events, MVD was between 104.9 and $342 / \mathrm{mm}^{2}$, with a mean of $154.7 / \mathrm{mm}^{2}$. In those who were free of events, it was between 3 and $203.7 / \mathrm{mm}^{2}$, with a mean of $45.2 / \mathrm{mm}^{2}$.

In the CHOP group, CD68 counts ranged from 80.6 to $452.2 / \mathrm{mm}^{2}$. In those without events, counts between 80.6 and $354.2 / \mathrm{mm}^{2}$ (mean: $233.3 / \mathrm{mm}^{2}$ ) were observed, and in those with events, counts between 19 and $452.2 / \mathrm{mm}^{2}$ (mean: $211.3 / \mathrm{mm}^{2}$ ) were observed.

The CD34 counts in this group were in the range of 19.8 to $623.2 / \mathrm{mm}^{2}$. The mean count in the patients with poor outcome was $349.2 / \mathrm{mm}^{2}$ (range: $228-623.2 / \mathrm{mm}^{2}$ ), whereas in those free of events, it was $56.6 / \mathrm{mm}^{2}$ (range: $19.8-121.6 / \mathrm{mm}^{2}$ ).

\section{Discussion}

Stromal-1 and stromal-2 signatures define the specific microenvironments contributed by the stromal cells in DLBCL. ${ }^{4}$ Tumor-associated macrophages (TAMs) are now proven to be present in the microenvironment of many hematological and nonhematological malignancies and are found to be associated with tumor progression, spread, and therapy resistance. ${ }^{8}$ TAMs are of different types. M1 subtypes (CD68-KP1 clone positive) are described to play a variable role in tumor regulation. Macrophage infiltration interferes with the tumor-associated inflammation and causes regression of tumor. ${ }^{9}$ The stromal- 1 signature as explained by Lenz et al is marked by genes such as MMP-9 and SPARC, which are associated with extracellular matrix deposition such as fibronectin, osteonectin, various collagens, laminin isoforms, and the antiangiogenic factor thrombospondin. These are also expressed in cells in the monocytic lineage. Thus, stromal-1 signature also signals a monocytic response in the microenvironment of lymphoma along with deposition of extracellular matrix. ${ }^{5}$

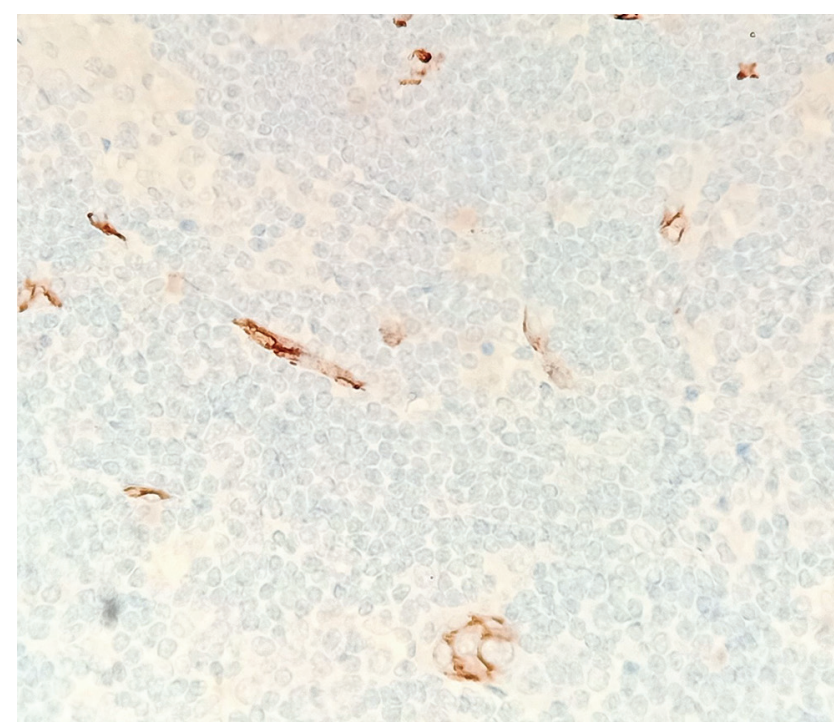

Fig. 1 Immunohistochemistry for CD34 highlighting microvessel density $\times 20$. 


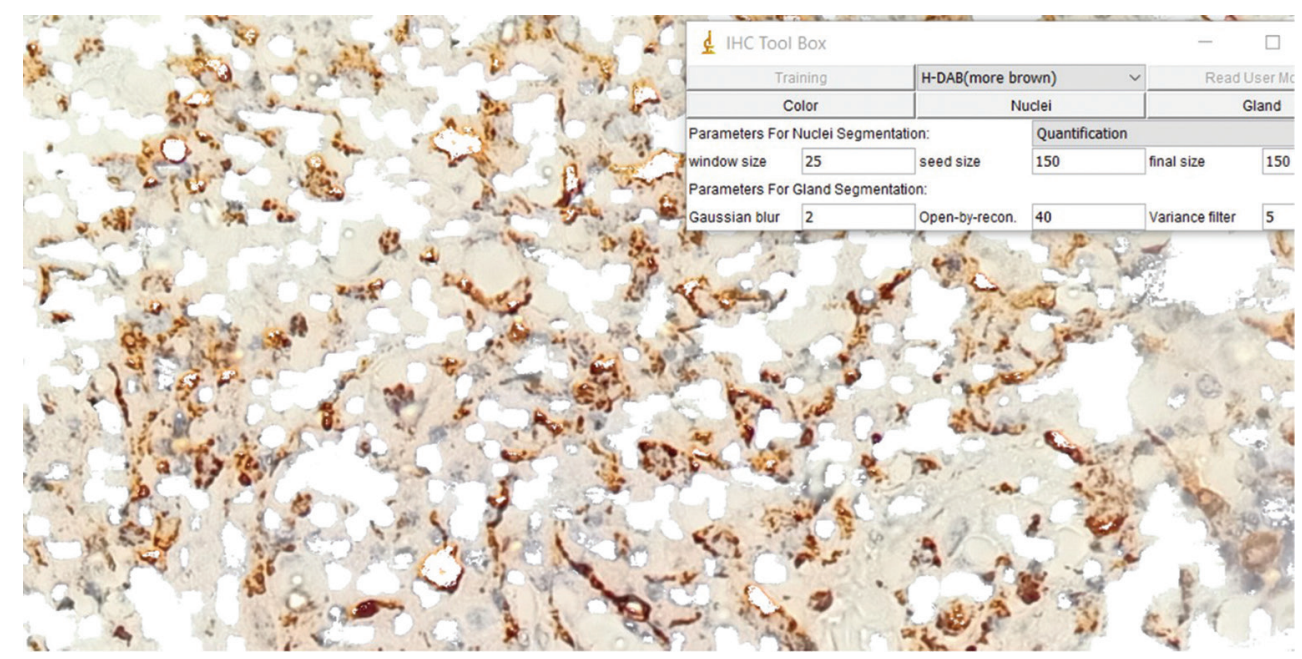

Fig. 2 Immunohistochemistry for CD68 along with the Image J analysis tool used for counting the positive cells.

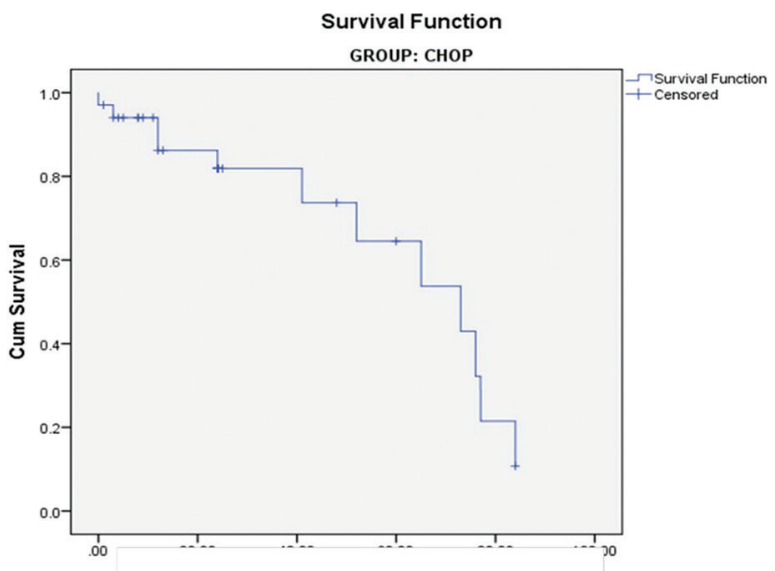

Fig. 3 Survival curve of patients treated with CHOP regimen.

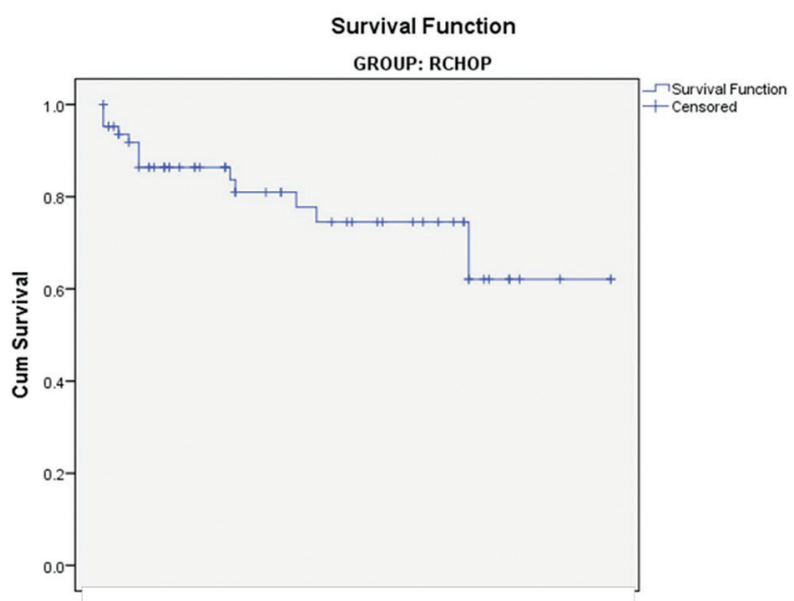

Fig. 4 Survival curve of patients treated with RCHOP regimen.
The M2 subtypes are supposed to promote tumorigenesis by altering the inflammatory environment by producing active substances such as reactive oxygen that induce oncogenesis. They can also activate programmed cell death protein 1 (PD-1) and programmed cell death-ligand 1 (PDL1), thereby inhibiting inflammation and immunity and promoting tumor cell growth. ${ }^{10}$

We studied the surrogate IHC markers CD68 and CD34 for stromal-1 and stromal-2 signatures in DLBCL, respectively, and correlated it disease outcome. There was a significant increase in the counts of CD68-positive cells among patients free of events (Student's $t$-test value: $5.4 ; p$ 0.00001) in the RCHOP group, with a mean count of $302.1 / \mathrm{mm}^{2}$.The median CD68 count in a study by Ruan et al was $231 / \mathrm{mm}^{2}$, and they stated that the higher the CD68-positive cell counts, the better the prognosis. ${ }^{11}$ Though the count in the event-free CHOP group was also high in our series, it was not statistically significant.

Pham et al found that those on RCHOP therapy were found to have a better prognosis when the CD68-positive cells in microenvironment was high. ${ }^{8}$ Cai et al reported poor outcomes associated with high CD68 count in CHOP-treated patients. The mechanism to be explored is the anti-CD20 antibody induced cytotoxicity or phagocytosis in the presence of macrophages. It is proposed that rituximab switches the protumor M2-type macrophage to antitumor M1 type. ${ }^{12}$ Others like Meyer et al. could not find any significant association of CD68 count with prognosis. ${ }^{13}$ This wide variation in observations may be due to the differences in the clone of the antibodies used, staining methods, and scoring systems, resulting in poor concordance.

The difference in the mean values of CD34-positive cell/ vessel counts (MVD) between these two groups (with and without events) was found to be statistically significant, with higher values in those with events, in both the CHOP and RCHOP arms (Student's t-test < 0.05). The mean MVD in the RCHOP group with events was $154.7 / \mathrm{mm}^{2}$ and that in 
the CHOP group was $349.2 / \mathrm{mm} .^{2}$ Median of CD34 was 37 in a study by Ruan et al. ${ }^{11}$ Our findings correlate with the study by Perry et al, who attempted to simulate the stromal-2 signature by measuring MVD in DLBCL and found high MVD to be an unfavorable prognostic factor. ${ }^{14}$

The stromal-2 signature is expressed by genes regulating endothelial proliferation through vascular endothelial growth factor (VEGF) receptor, TEK (tyrosine kinase, endothelial), and ERG V-ets (erythroblastosis virus E26 oncogene homo$\log$ gene). ${ }^{15}$ For all tumors to grow and survive, vascularity is essential. This needs angiogenic factors such as VEGF and FGF (fibroblast growth factor), which are induced by several mechanisms in a tumor, including hypoxia, oncogene upregulation, and tumor suppressor gene inhibition. Wide array of markers were studied to mark the endothelial cells, such as CD31, CD34, and von Willebrand factor. The study by Gomez-Gelvez et al showed that CD34 is the best stain owing to its clean background, and positive staining cells/cell groups with or without lumen formation represent microvessels. ${ }^{2}$ Therefore, we too did CD34 staining and counting using image analysis. MVD is the measure used to assess the angiogenesis in tumors including lymphomas. Stromal-2 signature thus shows high tumor blood vessel density, indicating more tumor angiogenesis, tumor survival, and poorer patient outcome.

Several studies have been conducted to analyze the reasons for heterogeneity in the behavior of DLBCL. Many risk stratification algorithms have been devised based on both tumor cell and their microenvironment characteristics so that appropriate first-line therapy can be delivered. The new paradigm shift in tumor biology suggests that the microenvironment of tumor plays a critical role in tumor immunology and growth by influencing the angiogenesis, and cell viability and motility, thus guiding the tumor spread and invasion.

Our study also emphasized the influence of tumor microenvironment on the behavior of DLBCL. High CD68-positive M1-type macrophage infiltration in the tumor was found to be associated with event-free survival in patients treated with both RCHOP and CHOP, though it was not statistically significant in the CHOP group. Similarly, high CD34 count (MVD) was found to have a significant association with poor outcome in both CHOP- and RCHOP-treated patients. Thus CD68 and CD34 can be considered as surrogate markers for stromal-1 and stromal-2 signatures, respectively, which can predict the treatment outcome in DLBCL patients.

\section{Conclusion}

Based on our analysis, we suggest that an additional assessment of stromal microenvironment along with the cell of origin might predict the clinical outcome better in DLBCL patients.

\section{Conflict of Interest}

None declared.

\section{References}

1 Suzumiya J. Current status and progress of lymphoma research in East Asian countries: Introduction and planning. Int J Hematol 2018;107(4):392-394

2 Gomez-Gelvez JC, Salama ME, Perkins SL, Leavitt M, Inamdar KV. Prognostic impact of tumor microenvironment in diffuse large B-cell lymphoma uniformly treated with R-CHOP chemotherapy. Am J Clin Pathol 2016;145(4):514-523

3 Bellas C, García D, Vicente Y, et al. Immunohistochemical and molecular characteristics with prognostic significance in diffuse large B-cell lymphoma. PLoS ONE 2014;9(6):e98169

4 Lenz G, Wright G, Dave SS, et al; Lymphoma/Leukemia Molecular Profiling Project. Stromal gene signatures in largeB-cell lymphomas. N Engl J Med 2008;359(22):2313-2323

5 Kridel R, Steidl C, Gascoyne RD. Tumor-associated macrophages in diffuse large B-cell lymphoma. Haematologica 2015;100(2):143-145

6 Pereira T, Dodal S, Tamgadge A, Bhalerao S, Tamgadge S. Quantitative evaluation of microvessel density using CD34 in clinical variants of ameloblastoma: an immunohistochemical study. J Oral Maxillofac Pathol 2016;20(1):51-58

7 Arena ET, Rueden CT, Hiner MC, Wang S, Yuan M, Eliceiri KW. Quantitating the cell: turning images into numbers with ImageJ. Wiley Interdiscip Rev Dev Biol 2017;6(2):e260

8 Pham LV, Pogue E, Ford RJ. The role of macrophage/B-cell interactions in the pathophysiology of B-cell lymphomas. Front Oncol 2018;8:147

9 Cioroianu AI, Stinga PI, Sticlaru L, et al. Tumor microenvironment in diffuse large B-cell lymphoma: role and prognosis. Anal Cell Pathol (Amst) 2019;2019:8586354

10 Nam SJ, Go H, Paik JH, et al. An increase of M2 macrophages predicts poor prognosis in patients with diffuse large B-cell lymphoma treated with rituximab, cyclophosphamide, doxorubicin, vincristine and prednisone. Leuk Lymphoma 2014;55(11):2466-2476

11 Ruan J, Hyjek E, Kermani P, et al. Magnitude of stromal hemangiogenesis correlates with histologic subtype of non-Hodgkin's lymphoma. Clin Cancer Res 2006;12(19):5622-5631

12 Cai QC, Liao H, Lin SX, et al. High expression of tumor-infiltrating macrophages correlates with poor prognosis in patients with diffuse large B-cell lymphoma. Med Oncol 2012;29(4):2317-2322

13 Meyer PN, Fu K, Greiner T, et al. The stromal cell marker SPARC predicts for survival in patients with diffuse large B-cell lymphoma treated with rituximab. Am J Clin Pathol 2011;135(1):54-61

14 Perry AM, Cardesa-Salzmann TM, Meyer PN, et al. A new biologic prognostic model based on immunohistochemistry predicts survival in patients with diffuse large B-cell lymphoma. Blood 2012;120(11):2290-2296

15 Gratzinger D, Zhao S, Marinelli RJ, et al. Microvessel density and expression of vascular endothelial growth factor and its receptors in diffuse large B-cell lymphoma subtypes. Am J Pathol 2007;170(4):1362-1369 